\title{
Urethral Reconstruction of Critical Defects in Rabbits Using Molecularly Defined Tubular Type I Collagen Biomatrices: Key Issues in Growth Factor Addition
}

\author{
Jody E. Nuininga, M.D., ${ }^{1, *}$ Martin J.W. Koens, M.Sc., ${ }^{2,}$ Dorien M. Tiemessen, B.Sc., ${ }^{1}$ \\ Egbert Oosterwijk, Ph.D., ${ }^{1}$ Willeke F. Daamen, Ph.D., ${ }^{2}$ Paul J. Geutjes, M.Sc., ${ }^{1}$ \\ Toin H. van Kuppevelt, Ph.D., ${ }^{2}$ and Wout F.J. Feitz, M.D., Ph.D.
}

Tubular type I collagen biomatrices with and without growth factors (GFs) were constructed and evaluated in a rabbit model for critical urethral defects. Porous tubular biomatrices with an inner diameter of $3 \mathrm{~mm}$ were prepared using highly purified collagen fibrils and were crosslinked with or without heparin. Heparinized biomatrices were supplemented with the heparin-binding GFs vascular endothelial GF, fibroblast GF-2, and heparin-binding epidermal GF. Biomatrices with and without GFs were used to replace a critical $1 \mathrm{~cm}$ urethral segment in rabbits $(n=32)$. All animals showed normal urination without urinary retention. General histology and immunohistology of graft areas $(2,4,12$, and 24 weeks after implantation) indicated that all biomatrices were replaced by urethra-like structures with normal appearing cytokeratin-positive urothelium surrounded by vascularized tissue. The GF-containing biomatrices showed an increase in extracellular matrix deposition, neovascularization, urothelium, glands, granulocytes, and fibroblasts, compared with biomatrices without GF. GFs substantially improved molecular features of healing but failed to be superior in functional outcome. Retrograde urethrography indicated a normal urethral caliber in case of biomatrices without GF, but a relative narrowing of the urethra at 2 weeks postsurgery and diverticula after 4 weeks in case of biomatrices with GF. In conclusion, tubular acellular type I collagen biomatrices were successful in repairing urethral lesions in artificial urethral defects, and inclusion of GF has a profound effect on regenerative processes.

\section{Introduction}

$\mathbf{R}$ EPAIR OF SEVERE HYPOSPADIAS is often associated with multiple clinical interventional procedures and grafting. Current grafting techniques include the use of donor tissues such as preputial skin or buccal mucosa. In most instances, the initial results are satisfactory. But long-term follow-up studies show unsatisfactory results and complications, as fistula formations, recurrent strictures, and necrosis can occur in up to $54 \%$ of patients. ${ }^{1,2}$ Additionally, the amount of donor tissue or tissue that is suitable for grafting can be a limiting factor. These issues may be relieved by tissue engineering strategies for urethral reconstruction. However, the development of an optimal biomaterial for urethral reconstruction remains a significant challenge and suitable constructs are currently not available in clinical practice. Biocompatible and biodegradable biomatrices have already been successfully applied for urethral repair, mostly as an onlay. ${ }^{3-5}$ In studies with tubular biomatrices, cellular seeding of the scaffold was an absolute requirement to achieve successful repair of the defect. ${ }^{6-9}$ These findings appear to suggest that the use of acellular matrices for urethral reconstruction is limited. Previously, we tubularized small intestinal submucosa (SIS) $\left(\mathrm{COOK}^{\circledR}\right)$ and molecularly defined biomatrices by suturing and compared the regenerative capacity in a rabbit urethral defect model. We reported equating results between these two scaffolds without noticeable complications. ${ }^{4}$ Construction of pretubularized type I collagen biomatrices and their supplementation with growth factors (GFs) may facilitate the surgical procedure

This article was published in an abstract form: Rabbit urethra replacement with a molecularly-defined tubular type I collagen biomatrix. J Ped Urol 5 Suppl 1, S18, 2009.

${ }^{1}$ Department of Urology, Pediatric Urology Centre, Radboud University Nijmegen Medical Centre, Nijmegen, The Netherlands.

${ }^{2}$ Department of Biochemistry, Radboud University Nijmegen Medical Centre, Nijmegen, The Netherlands.

*These authors contributed equally to this work. 
and improve cellular ingrowth, respectively. For urethral regeneration, multiple GFs are involved because different cell populations are needed to restore the reconstructed site. Epidermal GF (EGF) is known to play a key role in urothelial regeneration, ${ }^{10}$ whereas fibroblast GF-2 (FGF-2) and vascular endothelial GF (VEGF) are involved in angiogenesis and blood vessel maturation. ${ }^{11}$ As all three GFs bind to heparin, we developed a tubular collagenous biomatrix containing heparin and supplemented this with heparin-binding EGF (HB-EGF), VEGF, and FGF-2. Biomatrices with and without heparin and GFs were used to replace rabbit urethra and were evaluated using retrograde urethrography and immunohistochemical examination.

\section{Materials and Methods}

Unless stated otherwise, all chemicals were purchased from Merck.

\section{Preparation of tubular collagen biomatrices}

For the construction of tubular biomatrices, insoluble type I collagen fibrils were purified from pulverized bovine Achilles tendon as previously described. ${ }^{12}$ A $0.67 \mathrm{wt} \%$ type I collagen suspension in $0.25 \mathrm{M}$ acetic acid was prepared and incubated for $16 \mathrm{~h}$ at $4{ }^{\circ} \mathrm{C}$. Then the suspension was homogenized, deaerated by centrifugation at $250 \mathrm{~g}$ for $15 \mathrm{~min}$, and poured into polystyrene tubular moulds (all at $4^{\circ} \mathrm{C}$ ).
Stainless-steel mandrels (diameter $3 \mathrm{~mm}$ ) and spacers were placed. The molds were frozen at $-20^{\circ} \mathrm{C}$ for $4 \mathrm{~h}$ followed by lyophilization (Fig. 1A). The tubular collagen biomatrices were crosslinked with 1-ethyl-3-(3-dimethyl aminopropyl) carbodiimide (Fluka) and $N$-hydroxysuccinimide (Fluka). ${ }^{13,14}$ In brief, tubular biomatrices were incubated at a content of $0.4 \mathrm{mg}$ collagen $/ \mathrm{mL}$ in $50 \mathrm{mM}$ 2-morpholinoethane sulphonic acid (USB Corporation) containing 40\% (v/v) ethanol, $33 \mathrm{mM}$ 1-ethyl-3-(3-dimethyl aminopropyl) carbodiimide, and $6 \mathrm{mM}$ $\mathrm{N}$-hydroxysuccinimide ( $\mathrm{pH} 5.0$ ), with or without $0.25 \%(\mathrm{w} / \mathrm{v})$ heparin (Diosynth) for $4 \mathrm{~h}$ while shaking at $21^{\circ} \mathrm{C}$. Biomatrices were washed with $0.1 \mathrm{M} \mathrm{Na}_{2} \mathrm{HPO}_{4}, 1 \mathrm{M}$ and $2 \mathrm{M} \mathrm{NaCl}$, and demineralized water. Biomatrices were disinfected by $70 \%$ (v/v) ethanol washings and subsequently stored at $-20^{\circ} \mathrm{C}$. Before implantation, scaffolds were washed extensively with sterile phosphate-buffered saline (PBS, $\mathrm{pH} 7.4$ ) under sterile conditions.

For correct GF supplementation, simultaneous incubation (3.5 $\mu \mathrm{g} / \mathrm{mL}$ per GF), proportional incubation (5.5 $\mu \mathrm{g}$ FGF-2/ $\mathrm{mL}, 3.5 \mu \mathrm{g}$ VEGF/mL, $1.5 \mu \mathrm{g}$ HB-EGF/mL), and preincubation of FGF-2 were investigated. In the latter case, disinfected heparinized biomatrices $(4 \mathrm{mg}$ matrix $/ \mathrm{mL}$ ) were incubated in sterile PBS containing $3.5 \mu \mathrm{g} / \mathrm{mL}$ human recombinant FGF-2 (R\&D) for $1 \mathrm{~h}$ at $21^{\circ} \mathrm{C}$. Next, human recombinant VEGF (R\&D) and human recombinant HB-EGF (R\&D) were added (final concentration of $3.5 \mu \mathrm{g} / \mathrm{mL}$ PBS per GF) and incubated for $16 \mathrm{~h}$ at $21^{\circ} \mathrm{C}$. Subsequently, supplemented

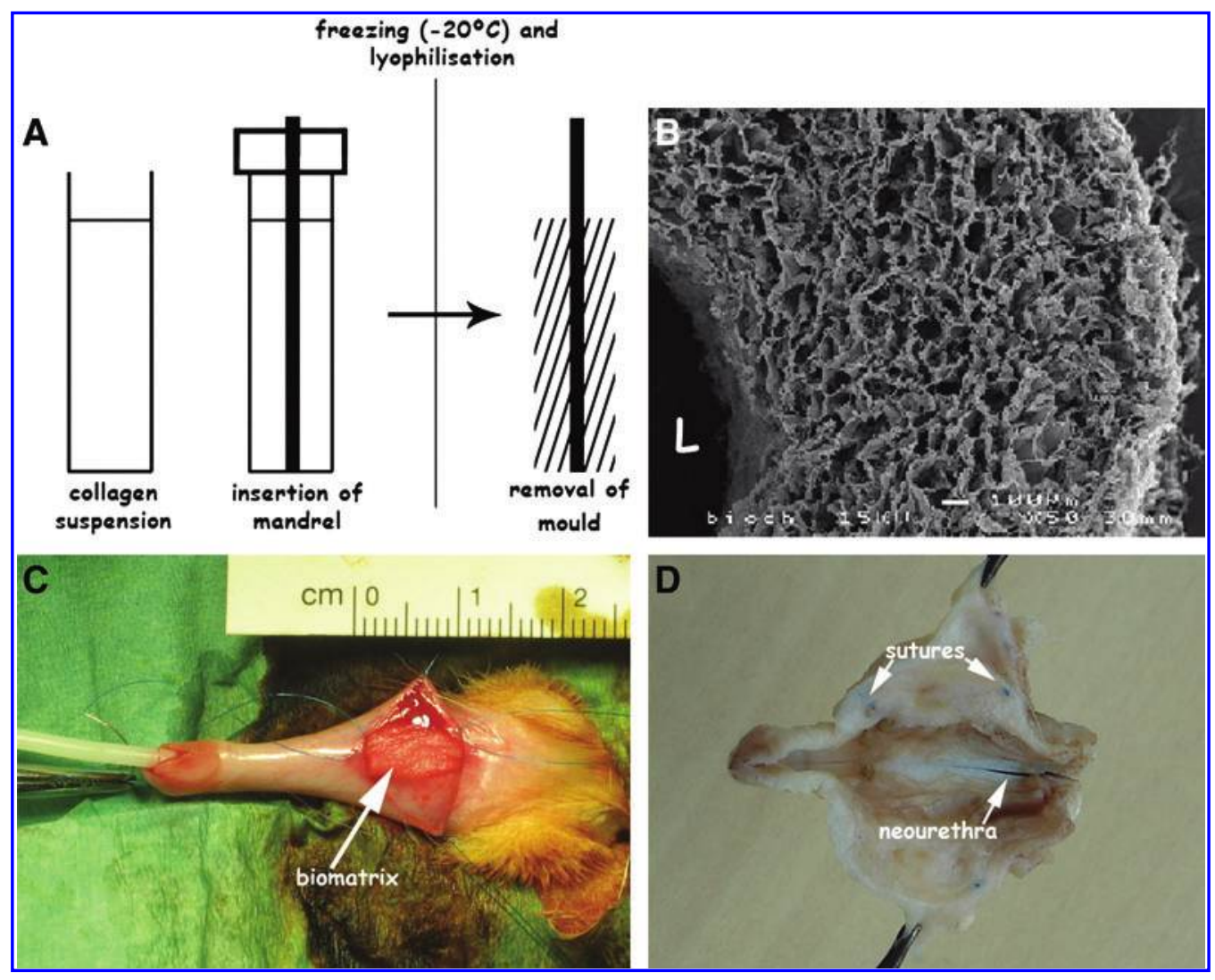

FIG. 1. Overview of the construction, implantation, and explantation of tubular type I collagen matrices. Scheme of the tubular molding process (A), a cross-sectional scanning electron microscopical image of part of a crosslinked tubular matrix (B, bar represents $100 \mu \mathrm{m}$ ), surgical replacement by a tubular matrix (arrow) of a $1 \mathrm{~cm}$ urethral defect (C), and explant at 24 weeks (D). L: lumen. Color images available online at www.liebertonline.com/ten. 
biomatrices were washed three times (each $15 \mathrm{~min}$ ) with sterile PBS and used immediately.

\section{Biochemical characterization of tubular collagen matrices}

The degree of collagen crosslinking was determined by a trinitrobenzosulfonic acid assay. ${ }^{12,15}$ The amount of bound heparin to the biomatrices was measured by hexosamine analysis. ${ }^{12}$ The bound GFs was determined using Western blot analysis. ${ }^{11,16}$ In short, biomatrix samples were run on sodium dodecyl sulfate-polyacrylamide gels, together with a series of known amounts of VEGF, FGF-2, and HB-EGF. Subsequently, gels were blotted, blocked using $1 \%$ bovine serum albumin (PAA), and incubated with either rabbit-antibovine FGF-2 (1: 8000; Sigma-Aldrich), goat-anti-human HBEGF (1:1000; R\&D), or goat-anti-rabbit VEGF (1:400; R\&D) containing $1 \%$ bovine serum albumin. After washing, blots were incubated with the appropriate secondary antibodies conjugated to horseradish peroxidase and washed. Peroxidase activity was detected by chemiluminescence using ECL ${ }^{\mathrm{TM}}$ Western Blotting Detection Reagents (GE Healthcare) followed by detection and digital quantification (Adobe Photoshop CS3).

\section{Scanning electron microscopy}

Scanning electron microscopy was performed to analyze the morphology of the biomatrices. The samples were fixed on a stub with double-sided carbon tape. Next, the samples were sputtered with an ultrathin gold layer and examined in a JEOL Scanning Electron Microscope 6310 at an accelerating voltage of $15 \mathrm{kV}$.

\section{Animals}

The animal experiment was approved by the Animal Ethics Committee of the Radboud University Nijmegen Medical Centre, Nijmegen, The Netherlands. A total of 32 male New Zealand white rabbits $(2.5-3.5 \mathrm{~kg})$ were housed individually in a temperature-controlled cage with 50\%-55\% humidity for a $12 \mathrm{~h}$ light-dark cycle with free access to standard laboratory chow and tap water. After a minimum of 2-week quarantine period, the rabbits were fasted overnight prior to surgery. No antibiotic prophylaxis was given. Animals received fentanyl-fluanisone $(0.5 \mathrm{~mL} / \mathrm{kg}$ intramuscularly) before induction of anesthesia.

\section{Surgical procedure for urethra replacement}

The surgical procedures have been described in detail elsewhere. ${ }^{4}$ In short, rabbits were placed on the operating table in the supine position and general anesthesia was achieved with $2 \%$ isofluorane by endotracheal intubation with spontaneous ventilation. Blood pressure, heart rate, and hemoglobin oxygen saturation were monitored continuously throughout the period of anesthesia. After shaving and washing of the ventral penis, the urogenital region was disinfected with povidone-iodine scrub. Next, a transurethral 8 F catheter was placed in situ. Urethrography was performed preoperatively. Under a 2.5 optical magnification, a complete resection of a urethral segment of $1 \mathrm{~cm}$ in length was made at $\sim 1.5 \mathrm{~cm}$ below the distal urethral meatus with the penis in stretch. A tubular biomatrix of $1 \mathrm{~cm}$ (diameter $3 \mathrm{~mm}$ ) was placed and secured with eight notched sutures $\left(7 \times 0\right.$ vicryl ${ }^{\circledR}$; Ethicon). Two nonresolvable sutures $\left(6 \times 0\right.$ prolene ${ }^{\circledR}$; Ethicon) were placed at the external ends of the tubular biomatrix for future reference. The subcutis and skin were closed in two layers with $7 \times 0$ vicryl suture. The transurethral catheter was removed directly after surgery.

\section{Explantation schedule}

All rabbits were euthanized with an overdose of pentobarbital. Then, urethrography and collection of the urethra was performed. The total penis was removed, fixed in $4 \%$ $(\mathrm{v} / \mathrm{v})$ formalin in PBS for $24 \mathrm{~h}$, and embedded in paraffin. The rabbits were divided into two groups: a crosslinked collagen biomatrix group (COLX) and a group that received heparinized tubular biomatrices supplemented with VEGF, FGF-2, and HB-EGF (COLX-Hep-GF). Four rabbits were sacrificed in each group at 2, 4, 12, and 24 weeks after implantation $(n=32)$.

\section{Immunohistological examination}

Macroscopic inspection and evaluation of the urethra was performed. For histological examination, two samples of every group were cut into four equal transversal pieces. The other two samples were processed longitudinally. Then, sections were cut $(4 \mu \mathrm{m})$ and stained with hematoxylin and eosin. Sections were scored independently by two panels of two investigators (J.N. and P.G., and M.K. and W.D.) for cellular infiltration, neovascularization, new extracellular matrix (ECM) formation, urothelium, and biomatrix

Table 1. Biochemical Characteristics of Biomatrices

\begin{tabular}{|c|c|c|c|c|c|c|}
\hline $\begin{array}{l}\text { Tubular } \\
\text { scaffolds }\end{array}$ & $\begin{array}{l}\text { Crosslinked } \\
\text { using } \\
\text { EDC/NHS }\end{array}$ & $\begin{array}{c}\text { Amine group content } \\
\text { (nmol/mg biomatrix) } \\
\mathrm{n}=3(\text { mean } \pm \text { s.d. })\end{array}$ & $\begin{array}{l}\text { Heparin content } \\
(\mu \mathrm{g} / \mathrm{mg} \text { biomatrix }) \\
\mathrm{n}=3(\text { mean } \pm \text { s.d. })\end{array}$ & $\begin{array}{c}\text { VEGF content } \\
(n g / m g \text { biomatrix }) \\
\mathrm{n}=4(\text { mean } \pm \text { s.d. })\end{array}$ & $\begin{array}{c}\text { FGF-2 content } \\
(n g / m g \text { biomatrix }) \\
\mathrm{n}=4(\text { mean } \pm \text { s.d. })\end{array}$ & $\begin{array}{c}\text { HB-EGF content } \\
(n g / m g \text { biomatrix }) \\
\mathrm{n}=4(\text { mean } \pm \text { s.d. })\end{array}$ \\
\hline COL & No & $306 \pm 16$ & $0^{\mathrm{a}}$ & - & - & - \\
\hline COLX & Yes & $189 \pm 10$ & - & - & - & - \\
\hline COLX-Hep-GF & Yes & $135 \pm 4$ & $178 \pm 15$ & $116 \pm 63$ & $104 \pm 6$ & $162 \pm 27$ \\
\hline
\end{tabular}

The amounts of amine groups (indicative for crosslinking), heparin, and various growth factors are given. COL were used as reference. aset to 0.

EDC, 1-ethyl-3-(3-dimethyl aminopropyl) carbodiimide; NHS, N-hydroxysuccinimide; s.d., standard deviation; VEGF, vascular endothelial growth factor; FGF-2, fibroblast growth factor-2; HB-EGF, heparin-binding epidermal growth factor; COL, noncrosslinked collagen biomatrices; COLX, crosslinked collagen biomatrices; COLX-Hep-GF, heparinized collagen biomatrices supplemented with growth factors; -, not determined. 
FIG. 2. Retrograde urethrograms. The urethrograms of the COLX group showed normal caliber of the urethra at 2, 4, 12, and 24 weeks after implantation $(\mathrm{A}, \mathrm{C}, \mathrm{E}, \mathrm{G})$. In the COLX-Hep-GF group $(\mathbf{B}, \mathbf{D}, \mathbf{F}, \mathbf{H})$, rabbits had relative narrowing of the urethra at 2 weeks and diverticula after 4 weeks. Arrows indicate the position of the biomatrix. COLX, crosslinked collagen biomatrix; COLX-Hep-GF, heparinized collagen biomatrices supplemented with growth factors.
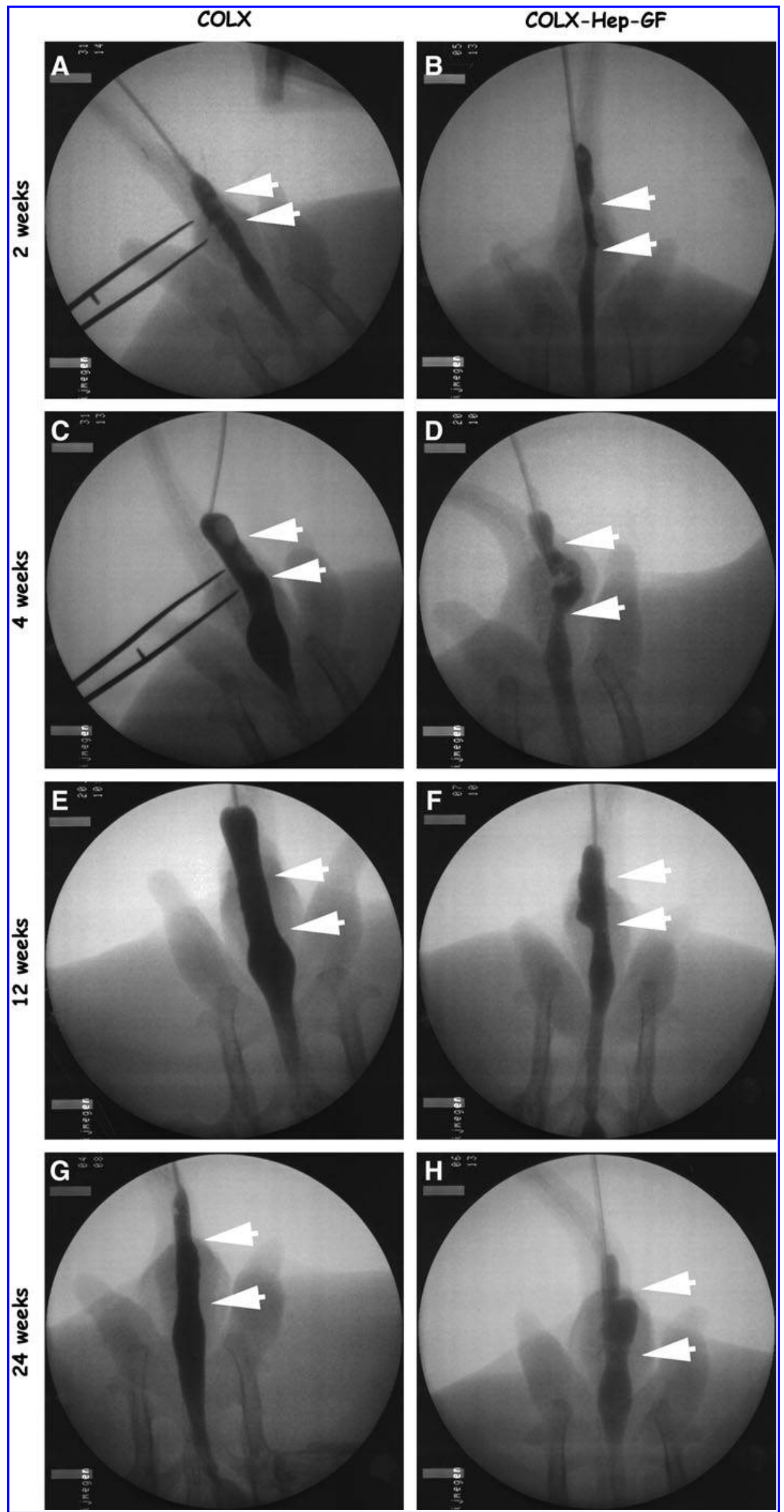
remnants. In case of dissimilar scores, slides were reevaluated until agreement was reached.

Immunohistochemistry was performed for CD31, pancytokeratin, desmin, and smooth muscle actin. In brief, paraffin sections were deparaffinized in xylene and hydrated in a degrading series of ethanol. After blocking of endogenous peroxidase activity, sections were incubated with monoclonal mouse anti-human CD31 (1:20; Dako), monoclonal mouse anti-human cytokeratin (1:800; LabVision), monoclonal mouse anti-human desmin (1:200; Biogenex), or monoclonal mouse anti-human smooth muscle actin $(1: 15,000$; Sigma). After PBS washings, sections were incubated with the appropriate peroxidase-conjugated secondary antibodies. After PBS washings, sections were developed with power diaminobenzidine (DAB) (Immunologic) as a chromogen and dehydrated and then slides were mounted with permount.

\section{Results}

\section{Biomatrix characterization}

Table 1 reviews the biochemical characteristics of the biomatrices. The biomatrices were highly porous (pores ranging 100-150 $\mu \mathrm{m}$; Fig. 1B) and successfully crosslinked as evidenced by a decrease of amine group content of $38 \%$ and $56 \%$ for the crosslinked and heparinized biomatrices, respectively. Heparinized biomatrices contained $18 \mathrm{wt} \%$ of heparin. Estimates from Western blot analyses revealed a total amount of $0.4 \mu \mathrm{g}$ bound GFs per milligram of scaffold (VEGF 30\%, FGF-2 27\%, HB-EGF 42\%).

The method used to bind GFs involved incubation of FGF-2 after which VEGF and HB-EGF were added. This method was found to be the most optimal method resulting in binding approximately equal amounts of each GF, as HBEGF was highly competitive in binding heparin, especially with respect to FGF-2. Other incubation methods, namely simultaneous or proportional incubation, were not adequate. The GF incubation concentrations were selected based upon a previous work. ${ }^{11}$

\section{Clinical observations}

All animals recovered satisfactory from the initial surgery (Fig. 1C) and survived without any sign of difficulty in urinating or urinary retention. No strictures or urethrocutaneous fistula were observed in the group without GFs (COLX) (Fig. 1D). The retrograde urethrogram of the group without GFs showed normal caliber of the urethra at all time points (Fig. 2A, C, E, F). Only two rabbits developed small diverticula at 12 weeks. In the group with heparin and GF-supplemented tubular biomatrices (COLX-Hep-GF) (Fig. 2B, D, F, H), all four rabbits showed relative narrowing of the urethra at 2 weeks (Fig. 2B). In 11 of 12 rabbits receiving COLX-Hep-GF tubular biomatrices $(4,12$, and 24 weeks postrepair groups), a diverticula of the urethra was observed. One rabbit in this group showed a urethrocutaneous fistula at 24 weeks (Fig. 2D). Macroscopic inspection confirmed these findings on urethrography.

\section{Histological examination}

The microscopical evaluation of urethral regeneration was semiquantified and is summarized in Table 2. Representative images are shown in Figures 3 and 4.
Table 2. Summary of Cellular Features, Listing Urothelium-Related Topics Separately

Weeks of implantation

2 weeks 4 weeks 12 weeks 24 weeks

\section{COLX}

Granulocytes

Lymphocytes

Giant cells

Macrophages

Neovascularization

Biomatrix remnants

ECM production

Fibroblast-like cells

Urothelium

Number of glands

Glandular hyperplasia

Budding

COLX-Hep-GF

Granulocytes

Lymphocytes

Giant cells

Macrophages

Neovascularization

Biomatrix remnants

ECM production

Fibroblast-like cells

Urothelium

Number of glands

Glandular hyperplasia

Budding

$\begin{array}{cccc}++ & + & - & - \\ \mathrm{sp} & \pm & \mathrm{sp} & \pm \\ \mathrm{sp} & ++ & \mathrm{sp} & \pm \\ \pm & \mathrm{sp} & - & \mathrm{sp} \\ \pm & \pm & ++ & ++ \\ ++ & ++ & - & - \\ \pm & + & ++ & ++ \\ \pm & + & ++ & ++ \\ \pm & \pm & ++ & ++ \\ - & - & ++ & +/++ \\ - & - & \pm & + \\ - & - & \pm & + \\ & & & \\ ++ & ++ & - & - \\ \mathrm{sp} & + & + & \mathrm{sp} / \pm \\ \pm & ++ & + & \pm \\ \pm & + & \pm & \mathrm{sp} \\ \pm & +/++ & ++ & ++ \\ ++ & ++ & + & \pm \\ + & +/++ & ++ & ++ \\ + & +/++ & ++ & ++ \\ \pm & +/++ & ++ & ++ \\ - & ++ & ++ & + \\ - & + & + & + \\ - & + & ++ & +\end{array}$

Scoring was done by two independent couples. Events were scored ranging from sporadic (sp) to abundant $(++)$.

COLX, crosslinked collagen biomatrix; ECM, extracellular matrix; COLX-Hep-GF, heparinized collagen biomatrices supplemented with growth factors; -, not present.

Two weeks evaluation. Granulocytes infiltrated the biomatrix, regardless of the biomatrix modification procedure with GFs (Fig. 3A, B). More giant cells were observed in the COLX-Hep-GF biomatrix. Neovascularization (CD31) was observed at the rim of the biomatrix (Fig. 4A, B). The COLXHep-GF biomatrix revealed purple-pink-colored matrix structures on hematoxylin and eosin staining, indicative of the presence of immobilized heparin. Despite precautions to completely remove the native urothelium, some remnants with smooth muscle cells underneath could be localized at the dorsal site of the urethra in almost all rabbits. This was confirmed by staining for desmin. Tissue regeneration was observed in the biomatrix as well as below the biomatrix. In this manner, the tubular construct also facilitated urethral reconstruction by functioning as an onlay.

Four weeks evaluation. The amount of granulocytes decreased in the COLX biomatrix but not in the COLX-HepGF biomatrix (Fig. 3C, D). More fibroblast-like cells and newly formed ECM were present in the COLX-Hep-GF biomatrix compared with COLX. As in the latter, still empty porous biomatrix structures could be found. The number of giant cells, present at the periphery of the biomatrix, was higher in COLX-Hep-GF compared with COLX. Also, neovascularization was increased in the COLX-Hep-GF group, 

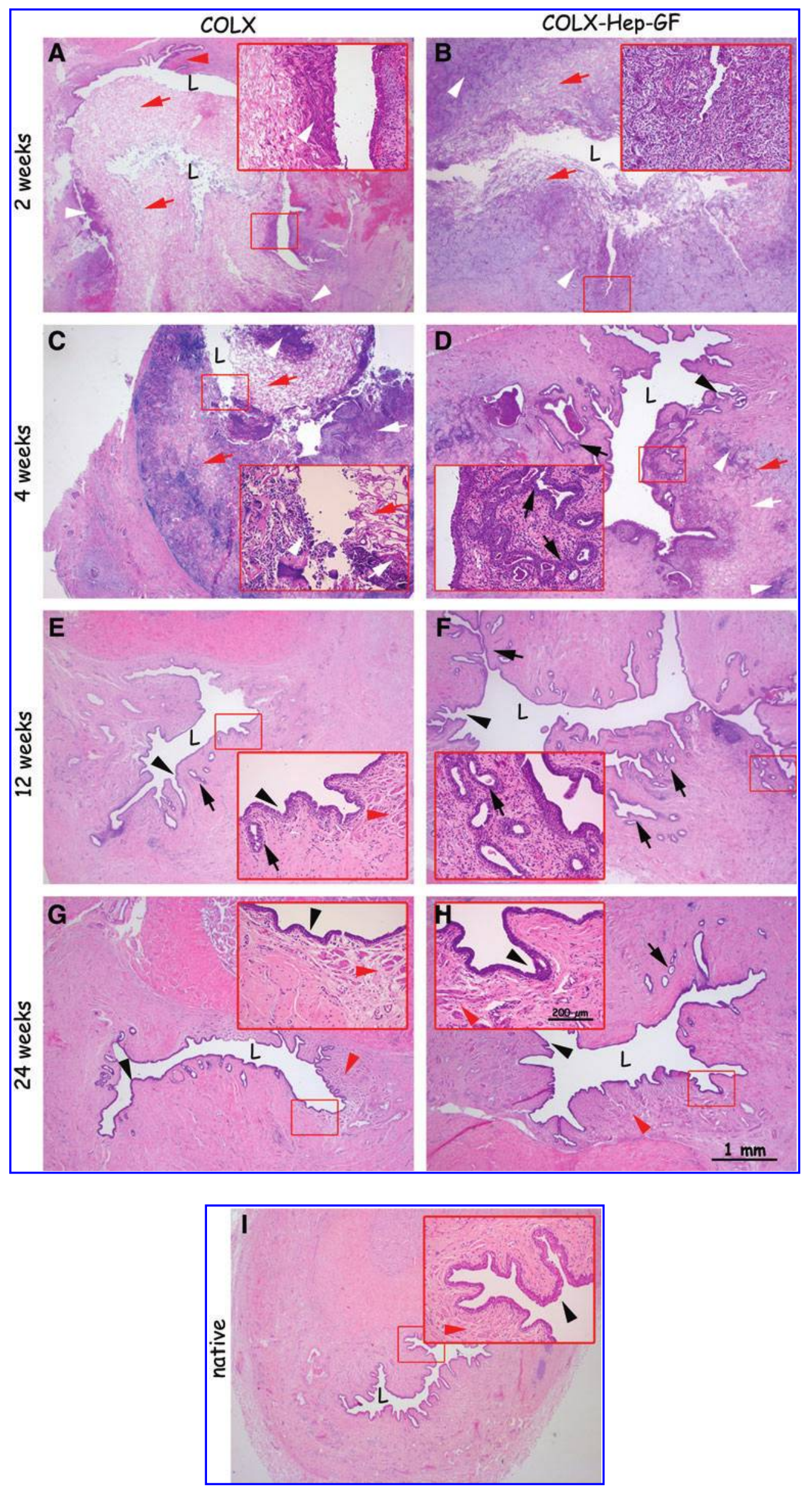
based upon more CD31-positive vessels. Newly formed and organized urothelium was cytokeratin positive and found to be increased in the COLX-Hep-GF biomatrix, though only sporadically present in the COLX biomatrix (Fig. 4C, D).

Twelve weeks evaluation. The cellular infiltration of granulocytes was reduced in all biomatrices (Fig. 3E, F). More lymphocytes and macrophages were present in the COLX-Hep-GF biomatrix in comparison to the COLX biomatrix. In both types of biomatrices, the level of interstitial ECM production and fibroblasts was increased in comparison to the situation at 4 weeks after implantation. In the COLX group, the scaffold was completely replaced by urethra-like tissue. In contrast, biomatrix remnants were still observed in the COLX-Hep-GF biomatrix. In both biomatrices, urothelium and capillaries became more evident. Neovascularization (CD31 staining) was comparable between both groups. A profound increase of glands, which were also positive for cytokeratin, and fusion of these was present in the COLX-HEP-GF in comparison to the COLX biomatrix (Fig. 4E, F).

Twenty-four weeks evaluation. The inflammatory response was additionally decreased. Both types of biomatrices were replaced by urethra-like structures; a normal transitional layer of epithelium was surrounded by vascularized tissue (Fig. 3G, H). COLX-Hep-GF biomatrices were almost fully degraded. A decrease in the number of glands was seen in both groups in comparison with 12 weeks after implantation (Table 2). Fusion of these, or budding, was increased in COLX and decreased in COLX-Hep-GF. No muscle cells were visible in the new tissue of both groups. A clear transition could be made between old and new tissue, as the latter was recognizable by underlying desmin-positive muscle (Fig. 4G, H). Both old and new tissues had cytokeratin-positive epithelium that was comparable to one another.

\section{Discussion}

Literature data suggest that cell seeding is critical to regenerate urethral tissue and avoid stricture formation when collagen grafts are used for urogenital reconstructions. ${ }^{6-9}$ Cell seeding necessitates tissue harvesting for the cellular components, causing an increase in morbidity, and-more importantly-comes with a complex, labor-intensive, and time-consuming procedure. It would be advantageous to develop an acellular biomatrix with a high capacity to regenerate urethral tissues. A tubular biomatrix may be a suitable alternative for patients in need for a total urethral reconstruction, such as patients with severe hypospadias or long urethral strictures. In a previous study, ${ }^{4}$ we investigated an onlay urethral procedure in a rabbit model with an unmodified, acellular collagen-based biomatrix, compared it with an SIS, and observed comparable regeneration of the urethra. Encouraged by the results of that study, we decided to develop novel, molecularly defined tubular biomatrices (containing pores of about 100-150 $\mu \mathrm{m}$ ) substituted with three GFs (VEGF, FGF-2, and HB-EGF), by coupling with covalently attached heparin. These GFs are known to have a stimulatory effect on various cellular processes including cell influx, angiogenesis, and proliferation/differentiation, thus improving the regenerative capacity of the construct. It is known from literature that epidermal GF plays a key role in urothelial regeneration, ${ }^{10}$ whereas FGF-2 and VEGF are involved in angiogenesis and blood vessel maturation. ${ }^{11}$ In an in vitro pilot study, optimal proliferation of urothelial cells (SCaBER) was seen by the sole use of EGF (unpublished results).

We evaluated the regenerative capacity of these biomatrices without and with GFs (COLX and COLX-Hep-GF) in a rabbit model by replacing $1 \mathrm{~cm}$ urethra, a critical-sized defect, ${ }^{17}$ and compared the outcome at 2, 4, 12, and 24 weeks after implantation. Successful regeneration without urethral occlusion was achieved with the tubular COLX biomatrix. Retrograde urethrogram analysis indicated normal caliber of the urethra at all time points, underscoring its effectiveness. Histology of the COLX biomatrix indicated a mild cellular response, with the formation of normal urothelium and capillaries within 24 weeks. For the COLX-Hep-GF biomatrix, a profound effect on tissue regeneration was observed, including an increase in ECM, urothelium, capillaries, glands, and the number of granulocytes, lymphocytes, and macrophages. Sievert et al. already showed the importance of the time-dependent changes in GF expression during regeneration. Through the addition of GF to the biomatrix we mimic a depot of GFs in a slow release system, leading to faster urethral regeneration. ${ }^{18}$ Additionally, genetically engineered urethral grafts expressing human VEGF significantly induced neovascularization in the urethral wall. ${ }^{19}$ VEGF attracts endothelial cells and the combination with FGF-2 has proven to further enhance an early and well-developed vasculature. ${ }^{11}$ Nevertheless, although we assumed that the addition of GF would facilitate and quicken urethral regeneration, urethrogram analysis of the COLX-Hep-GF group indicated a relative narrowing of the replaced urethral segment at 2 weeks and dilatation (diverticula) at 12 and 24 weeks. The histological analyses suggest that dilatation is caused by an increase in urothelium ingrowth in comparison to the unmodified biomatrix. The fact that FGF-2 and HB-EGF promote epithelial

FIG. 3. Histology of explanted biomatrices (hematoxylin and eosin staining). The left column displays results of the COLX group; the right column displays results of the COLX-Hep-GF group. The hematoxylin and eosin staining at the bottom shows the native tissue, with urothelium (black arrowhead) and the underlying smooth muscle (red arrowhead). (I) At week 2 after implantation, granulocytes had infiltrated the biomatrix (white arrowhead) (A, B). After 4 weeks, more newly formed extracellular matrix (white arrow) was present in the COLX-Hep-GF biomatrix in comparison to the COLX biomatrix. Remnants of the biomatrix (red arrow) were found in the neourethra in both groups (C, D). An increase of glands and an increase in fusion (black arrow) of these glands were observed in the COLX-Hep-GF in comparison to the normal COLX biomatrix at 12 weeks (E, F). Both types of biomatrices were replaced by urethra-like structures at 24 weeks. The neourethra was only distinguishable from normal urethra by the fact that there were no muscle cells visible in the replacement tissue (G, H). Black arrowheads show the border between native tissue and new regenerated tissue. Bars represent $1 \mathrm{~mm}$ and $200 \mu \mathrm{m}$ within the inset. L: lumen. Color images available online at www.liebertonline.com/ten. 


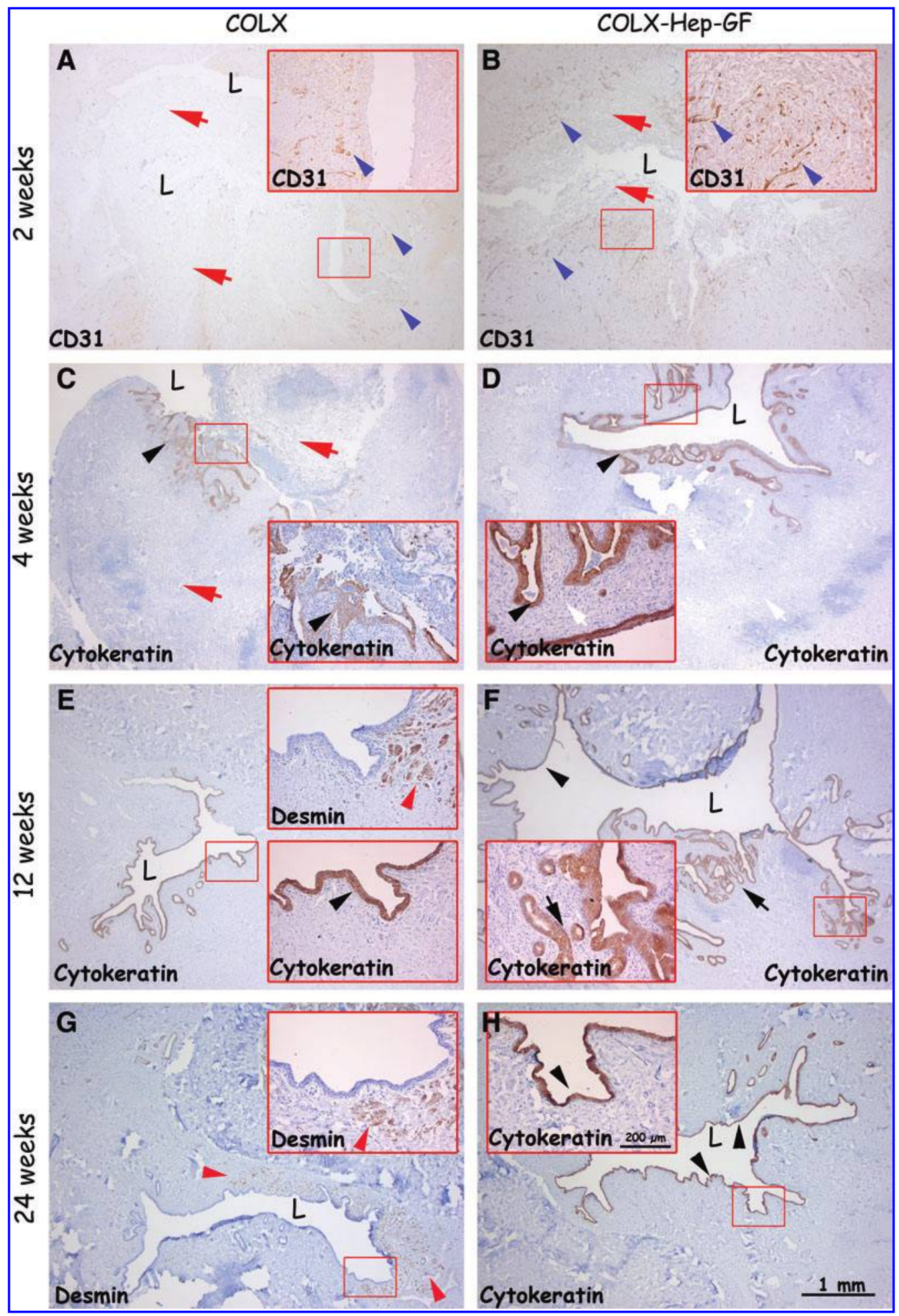

FIG. 4. Immunohistochemical analysis of blood vessels, urothelium, glands, and muscle cells in biomatrices. Neovascularization (CD31 staining, blue arrowheads) in the biomatrix at 2 weeks after implantation (A, B). Newly formed urothelium at 4 weeks after implantation (cytokeratin staining, black arrowheads), indicating a more organized urothelial layer in the COLX-Hep-GF biomatrix in comparison to the COLX biomatrix $(\mathbf{C}, \mathbf{D})$. This new urothelium also resulted in an increase and fusion of glands (black arrows) in the COLX-Hep-GF group in comparison to the normal COLX biomatrix (E, F, H). Both types of biomatrices were replaced by urethra-like structures and only distinguishable from normal urethra by the lack of smooth muscle cells in the newly formed tissue compared with original tissue (desmin staining, red arrowhead) (G). Bars represent $1 \mathrm{~mm}$ and $200 \mu \mathrm{m}$ within the inset. L: lumen; red arrow: biomatrix remnants; white arrow: newly formed extracellular matrix. Color images available online at www.liebertonline.com/ten. 
migration and reepithelialization in wound healing ${ }^{20,21}$ may explain the urothelium migration and proliferation into the wall of the biomatrix. This may be prevented by binding HBEGF only to the luminal side of the biomatrix wall. Epithelial glands that were formed inside the construct seemed to fuse into lacunae. This may explain the decrease in the absolute number of glands after 24 weeks. In a human setting, periurethral glands are of eminent importance; for example, glands of Littré are needed for a normal secretion of the seminal substance. Additionally, these glands are known to form, at times, small diverticula known as lacunae of Morgagni. ${ }^{2,23}$ These results implicate that the tubular collagen COLX biomatrix is capable of and succeeds in the regeneration of functional urethra-like tissue. The tubular COLX biomatrix had better functional outcome in comparison to tubular COLX-hep-GF biomatrix. GFs substantially improved "molecular" features of healing but failed to be superior in functional outcome (narrowed urethras, urethras with diverticula, and one fistula). The present study shows that caution should be regarded with respect to the use of GFs, and that biomatrices without GFs do not have to be inferior to those with GFs.

In binding GFs, the biomatrices were first bound with the glycosaminoglycan heparin. After 12 weeks, COLX-Hep-GF biomatrix remnants were still present, whereas the plain collagen biomatrix was fully degraded. This may be explained by the fact that the incorporated heparin is highly negatively charged because of the presence of sulfate and carboxylic groups, which may protect collagen from proteolysis, and has an inhibitory effect on the macrophage adhesion, thus delaying matrix degradation. ${ }^{24}$

Even though every precaution was taken to completely remove the native urothelium during surgery, this was extraordinarily difficult without causing excessive bleeding from the corpus cavernosa. As a result, some native urothelium was left at the dorsal site of the urethra. These urothelial remnants tend to induce tissue regeneration at the outside of the tubular construct, probably creating a new lumen through which urine was voided. As a result, the tubular construct most likely functioned as an onlay sometimes, thus implicating that at least small parts of the biomatrix could be lost because of voiding during (bio)matrix remodeling. The use of running sutures at the anastomoses or a temporary transurethral catheter may solve this problem.

Several investigators studied onlay urethroplasty in a rabbit model..$^{3-5}$ Single-layer SIS appears to be inferior to spontaneous urethral regeneration, possibly because SIS impedes smooth muscle cell regeneration, although results have been variable. ${ }^{3,7}$ The conflicting results might be the consequence of dissimilarities in different SIS batches. ${ }^{25}$

Similar to SIS, our constructs did not support smooth muscle cell ingrowth (smooth muscle actin). This is in contrast with results obtained with a decellularized tubularized construct created from SIS where complete regeneration of all urethral layers was reported as early as 12 weeks after urethra repair. ${ }^{3}$ However, the clinical importance and relevance of muscular cell ingrowth in a urethra is not clear. Urethroplasty with SIS resulted in fibrotic tissue covered by urethral mucosa without muscle cells at the site of SIS implantation. ${ }^{26}$ Animal studies have indicated that the urethra is capable of complete regeneration over a $15 \mathrm{~mm}$ defect area when only the ventral wall of the urethra is excised. ${ }^{7}$ However, a circumferential defect as used here does not heal spontaneously and this would imply that severe scarring may occur. Previous studies have shown that with an acellular tubularized construct the maximum defect distance that can be supported by normal tissue regeneration is $0.5 \mathrm{~cm}$, albeit that the longest time period studied was only 4 weeks. ${ }^{17}$

Collectively, in the present study, the repair of a $1 \mathrm{~cm}$ defect with collagen-based tubular biomatrices resulted in excellent regeneration of the urethra without urethral occlusion. In the future, long-term outcome of such matrices has to be studied. The inclusion of a support, for example, a polymer, may help to prevent voiding of urine alongside instead of through the construct.

\section{Conclusion}

Tubular, molecularly defined collagen biomatrices are successful in repairing urethral lesions of $1 \mathrm{~cm}$ in a rabbit model. The results with these developed biomatrices are promising, but further studies aimed at fine-tuning the effect of bound GFs must be performed, particularly to prevent (initial) excessive cell proliferation and to induce ingrowth of smooth muscle. Additionally, a composite construct may facilitate future use of longer tubular segments in urethral reconstruction.

\section{Acknowledgments}

Wilma Janssen-Kessels and Alex Hanssen from the Central Animal Facility Radboud University Nymegen Medical Centre (RUNMC) are gratefully acknowledged for their biotechnical assistance. Paul Jap is gratefully acknowledged for his histological expertise. The Microscopical Imaging Center (Nymegen Medical Centre for Life Sciences, Radboud University Nymegen Medical Centre [NCMLS, RUNMC]) is acknowledged for facilitating electron microscopy services. This study was financially supported by the Dutch program for Tissue Engineering (DPTE 6735), ZonMW 920-03-304, and EU-FP6 project EuroSTEC (soft tissue engineering for congenital birth defects in children; contract no.: LSHB-CT-2006-037409).

\section{Disclosure Statement}

No competing financial interests exist.

\section{References}

1. Sorber, M., Feitz, W.F.J., and de Vries, J.D.M. Short- and mid-term outcome of different types of one-stage hypospadias corrections. Eur Urol 32, 475, 1997.

2. Nuininga, J.E., de Gier, R.P.E., Verschuren, R., and Feitz, W.F.J. Long-term outcome of different types of 1-stage hypospadias repair. I Urol 174, 1544, 2005.

3. Kropp, B.P., Ludlow, J.K., Spicer, D., Rippy, M.K., Badylak, S.F., Adams, M.C., Keating, M.A., Rink, R.C., Birhle, R., and Thor, K.B. Rabbit urethral regeneration using small intestinal submucosa onlay grafts. Urology 52, 138, 1998.

4. Nuininga, J.E., Moerkerk van, H., Hanssen, A., et al. Rabbit urethra replacement with a defined biomatrix or small intestinal submucosa. Eur Urol 44, 266, 2003.

5. Chen, F., Yoo, J.J., and Atala, A. Acellular collagen matrix as a possible "off the shelf" biomaterial for urethral repair. Urology 54, 407, 1999.

6. Xie, H., Campbell, C.E., Shaffer, B.S., and Gregory, K.W. Different outcomes in urethral reconstruction using elastin and collagen patches and conduits in rabbits. J Biomed Mater Res B Appl Biomater 81, 269, 2007. 
7. El-Assmy, A., El-Hamid, M.A., and Hafez, A.T. Urethral replacement: a comparison between small intestinal submucosa grafts and spontaneous regeneration. BJU Int 94, 1132, 2004.

8. De Filippo, R.E., Yoo, J.J., and Atala, A. Urethral replacement using cell seeded tubularized collagen matrices. J Urol 168, 1789, 2002.

9. Fu, Q., Deng, C.L., Liu, W., and Cao, Y.L. Urethral replacement using epidermal cell-seeded tubular acellular bladder collagen matrix. BJU Int 99, 1162, 2007.

10. Daher, A., De Boer, W.I., El-Marjou, A., van der Kwast, T., Abbou, C.C., Thiery, J.P., Radvanyi, F., and Chopin, D.K. Epidermal growth factor receptor regulates normal urothelial regeneration. Lab Invest 83, 1333, 2003.

11. Nillesen, S.T., Geutjes, P.J., Wismans, R., Schalkwijk, J., Daamen, W.F., and van Kuppevelt, T.H. Increased angiogenesis and blood vessel maturation in acellular collagen-heparin scaffolds containing both FGF2 and VEGF. Biomaterials 28, 1123, 2007.

12. Pieper, J.S., Oosterhof, A., Dijkstra, P.J., Veerkamp, J.H., and van Kuppevelt, T.H. Preparation and characterization of porous crosslinked collagenous matrices containing bioavailable chondroitin sulphate. Biomaterials 20, 847, 1999.

13. Pieper, J.S., Hafmans, T., Veerkamp, J.H., and van Kuppevelt, T.H. Development of tailor-made collagenglycosaminoglycan matrices: EDC/NHS crosslinking, and ultrastructural aspects. Biomaterials 21, 581, 2000.

14. Olde Damink, L.H., Dijkstra, P.J., van Luyn, M.J., van Wachem, P.B., Nieuwenhuis, P., and Feijen, J. Cross-linking of dermal sheep collagen using a water-soluble carbodiimide. Biomaterials 17, 765, 1996.

15. Buttafoco, L., Engbers-Buijtenhuijs, P., Poot, A.A., Dijkstra, P.J., Daamen, W.F., van Kuppevelt, T.H., Vermes, I., and Feijen, J. First steps towards tissue engineering of smalldiameter blood vessels: preparation of flat scaffolds of collagen and elastin by means of freeze drying. J Biomed Mater Res B Appl Biomater 77, 357, 2006.

16. Burnette, W.N. "Western blotting": electrophoretic transfer of proteins from sodium dodecyl sulfate-polyacrylamide gels to unmodified nitrocellulose and radiographic detection with antibody and radioiodinated protein A. Anal Biochem 112, 195, 1981.

17. Dorin, R.P., Pohl, H.G., De Filippo, R.E., Yoo, J.J., and Atala, A. Tubularized urethral replacement with unseeded matri- ces: what is the maximum distance for normal tissue regeneration? World J Urol 26, 323, 2008.

18. Sievert, K.D., Bakircioglu, M.E., Nunes, L.,Tu, R., Dahiya, R., and Tanagho, E.A. Homologous acellular matrix graft for urethral reconstruction in the rabbit: histological and functional evaluation. J Urol 163, 1958, 2000.

19. Guan, Y., Ou, L., Hu, G., Wang, H., Xu, Y., Chen, J., Zhang, J., Yu, Y., and Kong, D. Tissue engineering of urethra using human vascular endothelial growth factor genemodified bladder urothelial cells. Artif Organs 32, 91, 2008.

20. Ono, I., Tateshita, T., and Inoue, M. Effects of a collagen matrix containing basic fibroblast growth factor on wound contraction. J Biomed Mater Res (Appl Biomater) 48, 621, 1999.

21. Inoue, M., Ono, I., Tateshita, T., Kuroyanagi, Y., and Shioya, N. Effect of a collagen matrix containing epidermal growth factor on wound contraction. Wound Repair Regen 6, 213, 1998.

22. Gillenwater, J.Y., Grayhack, J.T., Howards, S.S., and Duckett, J.W. Adult and Pediatric Urology, 3rd edition. St. Louis, MO: Mosby, 1996.

23. Wein, A.J., Kavoussi, L.R., Novick, A.C., Partin, A.W., and Peters, C.A. Campbell-Walsh Urology, 9th edition. Philadelphia, PA: Saunders, 2007.

24. Smetana, K., Jr. Macrophage recognition of polymers: effect of carboxylate groups. J Mater Sci: Mater Med 4, 526, 1993.

25. Kropp, B. Editorial comment. J Urol 170, 1578, 2003.

26. Hauser, S., Bastian, P.J., Fechner, G., and Muller, S.C. Small intestine submucosa in urethral stricture repair in a consecutive series. J Urol 68, 263, 2006.

Address correspondence to: Jody E. Nuininga, M.D. Department of Urology Pediatric Urology Centre Radboud University Nijmegen Medical Centre P.O. Box 9101 6500 HB Nijmegen

The Netherlands

E-mail: j.nuininga@uro.umcn.nl

Received: January 28, 2010

Accepted: June 01, 2010

Online Publication Date: July 21, 2010 


\section{This article has been cited by:}

1. Martin Vaegler, Andrew T. Lenis, Lisa Daum, Bastian Amend, Arnulf Stenzl, Patricia Toomey, Markus Renninger, Margot S. Damaser, Karl-Dietrich Sievert. 2012. Stem cell therapy for voiding and erectile dysfunction. Nature Reviews Urology . [CrossRef]

2. Hiroshi Mikami, Go Kuwahara, Nobuyuki Nakamura, Masayuki Yamato, Masatoshi Tanaka, Shohta Kodama. 2012. TwoLayer Tissue Engineered Urethra Using Oral Epithelial and Muscle Derived Cells. The Journal of Urology . [CrossRef]

3. Paolo Caione, Renata Boldrini, Annamaria Salerno, Simona Gerocarni Nappo. 2012. Bladder augmentation using acellular collagen biomatrix: a pilot experience in exstrophic patients. Pediatric Surgery International . [CrossRef]

4. Willeke Daamen, Kaeuis Faraj, Martin Koens, Gerwen Lammers, Katrien Brouwer, Peter Uijtdewilligen, Suzan Nillesen, Luc Roelofs, Jody Nuininga, Paul Geutjes, Wouter Feitz, Toin van KuppeveltExtracellular Matrix-Based Scaffolds from Scratch 385-398. [CrossRef]

5. Chao Feng, Yue-min Xu, Qiang Fu, Wei-dong Zhu, Lei Cui . 2011. Reconstruction of Three-Dimensional Neourethra Using Lingual Keratinocytes and Corporal Smooth Muscle Cells Seeded Acellular Corporal Spongiosum. Tissue Engineering Part A 17:23-24, 3011-3019. [Abstract] [Full Text HTML] [Full Text PDF] [Full Text PDF with Links]

6. Felix Wezel, Jennifer Southgate, David F.M. Thomas. 2011. Regenerative medicine in urology. BJU International no-no. [CrossRef]

7. Dr. Chao Feng , Dr. Yue-min Xu , Dr. Qiang Fu , Dr. Wei-dong Zhu, Dr. Lei Cui . Reconstruction of 3D neo-urethra using lingual keratinocytes \& corporal smooth muscle cells seeded acellular corporal spongiosum. Tissue Engineering Part A 0:ja. . [Abstract] [Full Text PDF] [Full Text PDF with Links] 